\title{
Green synthesis of Ag-Nps using Chrysopogon Zizanioide and Ocimum Sanctum Extract and its Antibacterial Activity
}

\author{
Punithavathi Manogaran, Suriyavathana Muthukrishnan, Kavitha Rani Mari, Anandhi \\ Eswaran
}

\begin{abstract}
For ecological synthesis of Ag-Nps, aqueous form of Chrysopogon zizanioid (S1), Ocimum sanctum (S2) and combined specimens (S3) is used as a reduction agent.The nanoparticles were synthesized and explained utilizing $X$-ray diffraction (XRD), FTIR and $U V$-vis, analysis. XRD model and Scanning Electron Microscopy (SEM) described the crystallinity of the Ag-Nps. Bi-molecules accountable for capping are a variety of Ag-Nps has been introduced by the FTIR spectral analysis. $\mathrm{UV}$-vis spectrum explored the feature of Ag-Nps synthetic. The Ag nanoparticles were using of a regular size of 7-15 $\mathrm{nm}$ and often spherical exact by XRD pattern. Synthesized Ag-Nps anti-bacterial action was assessed using diffusion technique with aqueous Chrysopogon zizanioid (S1), Ocimum sanctum (S2), and combined specimens (S3).The S3 sample Ag-Nps prominently reserved bacterial proliferation against multi-drug susceptible to the total pathogens used in this research.Thus, Ag-Nps shows a wide variety of reduced concentration antibacterial activity, which may be an excellent natural therapy choice in the future.
\end{abstract}

Index Terms: Chrysopogon zizanioide, Ag-Nps, Ocimum sanctum, XRD, SEM, FTIR

\section{INTRODUCTION}

Nanotechnology is an significant area of current studies on the development, approach and execution of particle orientation varying from approximately 1 to $100 \mathrm{~nm}$ in magnitude. Within this size spectrum, both atoms / molecules and their consequent size have altered the biological, physical and chemical characteristics. Due to their completely fresh or enhanced characteristics depending on their size and morphology, novel exploitation of nanoparticles and non-materials is growing rapidly on various fronts[1]. The advancement of green synthesis procedure for the mixture of nanoparticles is forming into a critical part of nanotechnology [2].For the past two centuries, the presently synthesized non-materials and their depiction are a nanotechnology sector that is inferable from their extensive utilization in certain areas[3].Various methods used to synthesize an Ag-Nps, including silver ion chemical reduction in water solution, include or exclude stabilizing thermal decomposition in organic solvents, chemical decrease and reducing photos in inverse micells, and chemical radiation reduction [4].Most of these techniques are particularly expensive and involve the use of poisonous, hazardous chemicals with the same harmful

\footnotetext{
Revised Manuscript Received on July 22, 2019.

Punithavathi Manogaran,

Suriyavathana Muthukrishnan,

Kavitha Rani Mari,

Anandhi Eswaran
}

ecosystem and biology.In comparison to the synthetic and physical experiment, biological techniques of nanoparticles synthesis using plant fungus, microbes, and enzymes were given as likely ecological choice [5,6].Sometimes by reducing the intricate method of keeping microbial populations, the combination of nanoparticles using plants can show remuneration over other biological processes [7].

In present time combination of AgNPs has concerned imperative owing to their various properties such as catalysis, optical and magnetic and antimicrobial properties [8,9]. Because of its rapid, non-pathogenic, ecological,economical operation, plant functions as a cause of green Ag-Nps synthesis have been taken into account and provide biosynthetic experiments used as a single phase method. Reducing and stabilizing silver ions through a combination of metabolites previously established in the therapeutic properties of plant extracts.

Various data is available on the exploitation of organic resources such as fungi, bacteria,yeast, honey and plants for Ag-Nps synthesis. In extension of the efforts for synthesizing AgNPs by green route, previous studies recorded the Ag-Nps synthesis from single plants but, this research introduces a description of the easy, quick and single-pot aqueous biosynthesis of such nanoparticles utilizing the combination of two extracts such as extract of Ocimum sanctum and extracts of Chrysopogon zizanioide.

\section{MATERIALS AND METHODS}

Experimental. All chemicals were using in this research were of high analytical quality and used without further purification. In this investigation, silver nitrate $\left(\mathrm{AgNO}_{3}\right.$, 99.9\%) was used. Through the synthesis Milli-Q water was utilized to ready all the aqueous solution.

Preparation of the plant extracts.

At the beginning, the roots of Chrysopogon zizanioid were methodically washed with double distilled water, accompanied by Milli-Q water.The roots were then sliced into smaller pieces. Heating $100 \mathrm{~g}$ of roots made the extract.The preparation extract has been placed in $500 \mathrm{ml}$ of Milli-Q water in Erlenmeyer flask for 30 minutes and it uses $\sim 100^{\circ} \mathrm{C}$ water bath. The extract was cooled to room temperature and filtered through a four-fold muslin cloth to obtain clear filtrate of the Chrysopogon zizanioid (S1) root extracts. At the initial phase, the aerial sections of Ocimum sanctum were washed carefully with a copious amount of double distilled water, accompanied by Milli-Q water.

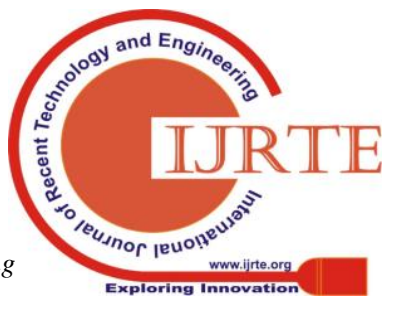


After slicing the leaves into pieces. The extract was prepared in Erlenmeyer flask using $\sim 100^{\circ} \mathrm{Cwater}$ bath by boiling $100 \mathrm{~g}$ of aerial components spread in $500 \mathrm{ml}$ of Milli-Q water for 30 minutes. The extract was cooled to room temperature and filtered for clear filtration of root extracts from Ocimum sanctum (S2) via a four-fold muslin cloth. After that $100 \mathrm{ml}$ from both aerial part extract of Ocimum sanctum and root extract of Chrysopogon zizanioide were mixed well and utilized for more analysis $\left(\mathrm{S}_{3}\right)$.

Ag-Nano particles synthesis from extract. Biosynthesis of Ag-Nanoparticles using aqueous plant sample of Chrysopogon zizanioid (S1), Ocimum sanctum (S2) and mixed samples (S3) was performed by adding $12 \mathrm{ml}$ of S1, S2 and $\mathrm{S} 3$ sample to an $88 \mathrm{ml}$ of $1 \mathrm{mM} \mathrm{AgNO}_{3}$ reaction mixture. The Ag-Nps prepared from the extract of the leaves and the supernatant were initially separated for $25 \mathrm{~min}$ by centrifugation at $10,000 \mathrm{rpm}$, accompanied by centrifugation at $15,000 \mathrm{rpm}$ for $30 \mathrm{~min}$. The pellets were after that with Milli-Q.water. The pellets were collected and used as an antibacterial activity against pathogens for further clarification and evaluation.

Characterization of Ag-Nps. Measurements of X-ray diffraction (XRD) were performed on Phillips PW 1830 devices operating at a radiation voltage of $40 \mathrm{kV}$ and a current of $20 \mathrm{~mA}$ with $\mathrm{CuK} \alpha$ radiation $(\pi=1,5406 \AA)$. On the Nicolet 6700 FTIR spectrometer, Fourier transforms infrared (FTIR) spectra were analyzed at room temperature. A small number of Ag-Nps as $0.01 \mathrm{~g}$ was dried at $60^{\circ} \mathrm{C}$ for $4 \mathrm{~h}$ for the FTIR trials of capped $\mathrm{Ag}-\mathrm{Nps}$ was mixed with $\mathrm{KBr}$ to create a round disk appropriate for FTIR readings.A appropriate quantity of the extract was mixed with $\mathrm{KBr}$ to obtain the extract's FTIR spectrum. The synthesized AgNPs were morphologically and chemically analyzed using an EDX detector fitted with JEOL 7001F FEG-SEM (REEQ/711/CTM/2005). UV-visible spectroscopic analysis was performed using UV-vis UV-1800 (Shimadzu) spectrophotometer.

Bacterial strains and cultivation. Bacterial strains like $S$. haemolyticus, Escherichia coli, Aeromonas hydrophila, Cronobacter sakazakii, Aeromonas salmonicida, and Basillus subtilis were used for experiment. In the $250 \mathrm{ml}$ conical flask, $50 \mathrm{ml}$ of LB broth was prepared and the bacterial strains cultivated on the orbital shaker in this environment at $370^{\circ} \mathrm{C}$. The culture flask was inoculated in the same culture conditions at 0.1 OD $600 \mathrm{~nm}$ with freshly prepared LB medium. The bacterial cultures of the mid-log phase were used for antibacterial research.

Disk diffusion method. In this technique overnight grown bacterial broth cultures containing 0.1 OD was inoculated on the $25 \mathrm{ml}$ of LB agar plates. Then the disk of whatman was put on the plates. Around $30 \mathrm{ul}$ of $\mathrm{S}_{1}, \mathrm{~S}_{2}$ and $\mathrm{S}_{3}$ samples were include on that whatman disc and incubate for overnight at $37^{\circ} \mathrm{C}$. Streptomycin was utilized.

\section{Results and discussion}

\section{X-ray diffraction (XRD)}

Fig.1 demonstrates patterns of synthesized Ag-Nps X-ray diffraction (XRD) at room temperature using extracts $S 1, S 2$ and S3. The Ag-Nps extract's XRD patterns depict the face-centered cubic (icc) structure of Ag-Nps. The XRD peaks would then be denoted to the $111,200,220,311$ crystallographic aircraft at 2 of $38.103^{\circ}, 44.31^{\circ}, 64^{\circ}$ and $77^{\circ}$ (JCPDS, section no.04-0783).The reflections (2 0 0), (2 20 ), and $\left(\begin{array}{lll}3 & 1 & 1\end{array}\right)$ of the Bragg are small and largely coupled with the intense reflection (1 1 ). This characteristic shows that $\left(\begin{array}{ll}1 & 1\end{array}\right.$ 1)-oriented nanocrystals are checked by high-resolution SEM measurements[10 ].Besides, the Bragg peaks denote fcc silver nanocrystals, unassigned peaks are also noted and bio-organic stage crystallization occurred on the nanoparticles surface.In Ag-Nps produced with geranium leaf extract and mushroom extract, similar findings have been reported, and maximum sharpness shows that nanoparticles have been introduced in nanoregime[11,12 ].It has been theoretically demonstrated that some other groups may be worried with bio-reduction of silver ions, i.e. amino acids, proteins and enzymes[13 ].This outcome indicates the greatest peak intensity in S3 associated with $\mathrm{S} 1$ and S2, and therefore it is obvious from the XRD pattern that Ag-NPs produced using all the extracts were essentially crystalline. By using well-known Debye-Scherrer formula, the size of average nanocrystalline was estimated,

$$
\mathrm{D}=(\mathrm{k} \lambda) /(\beta \cos \theta)
$$

Where $\mathrm{D}$ is a particle diameter size and $\mathrm{k}$ is an equal constant of 1 and $\mathrm{k}$ is an X-ray source wavelength $(0.1541 \mathrm{~nm}), \beta$ is a complete width at half maximum (FWHM) and $\mathrm{h}$ is a lattice plane diffraction angle (111).According to the calculated Debye-Scherrer equation, the average crystallite size for $\mathrm{S} 1$, $\mathrm{S} 2$ and S3 samples is $15 \mathrm{~nm}, 11 \mathrm{~nm}$ and $7 \mathrm{~nm}$ respectively, slightly higher than the particle size found in the Ag-NP TEM imageThis could be an indication of the slight variation of the particle spherical form that is crucial for the Debye-Scherrer formula.

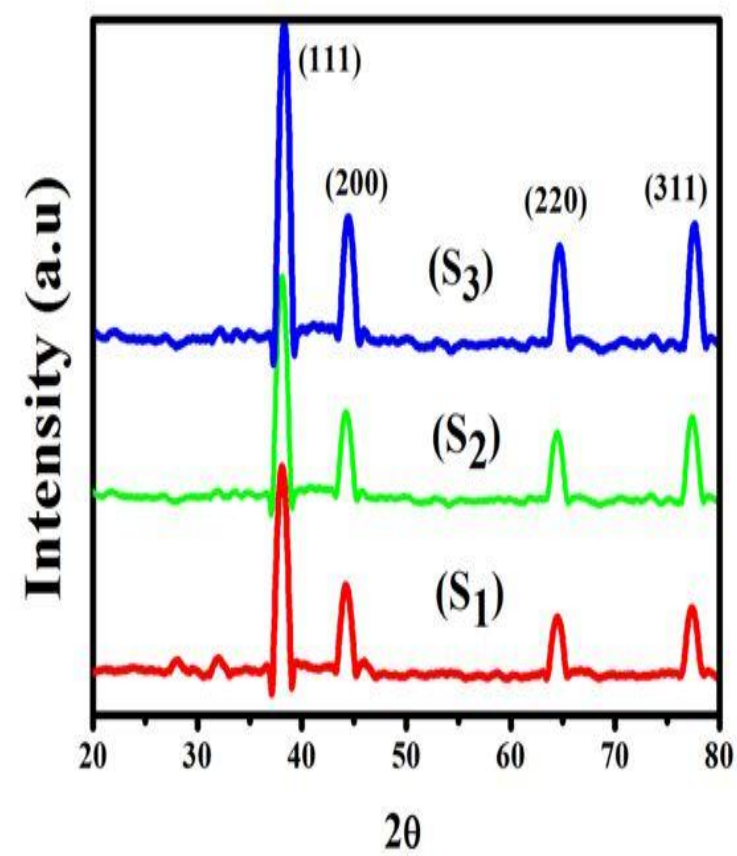

Fig. 1. X-ray diffraction pattern of Ag nanoparticles produced from $\mathrm{S} 1, \mathrm{~S} 2$ and $\mathrm{S} 3$ aqueous extract

FTIR Analysis. FTIR readings were done to identify the potential biomolecules required for capping in S1, S2 and S3 samples contributing to the effective stability of an Ag-Nps. FTIR spectrum of the Ag nanoparticles synthesized using $S_{1}$, $\mathrm{S}_{2}$ and $\mathrm{S}_{3}$ samples extracts are displayed in (Fig. 2), which displayed absorption peaks at 3777, 3395, 3285, 2924, 2855 , 1643, 1541, 1238, 1030, 780, 618, 566 and $466 \mathrm{~cm}^{-1}$. Between them, bands at $3200-3600 \mathrm{~cm}^{-1}$ display the typical signal for the symmetric and asymmetric stretching of the $\mathrm{O}-\mathrm{H}$ groups. The absorption peak at $1643 \mathrm{~cm}^{-1}$ shows the existence of $\mathrm{C}=\mathrm{O}$ groups in extracts and $\mathrm{C}=$

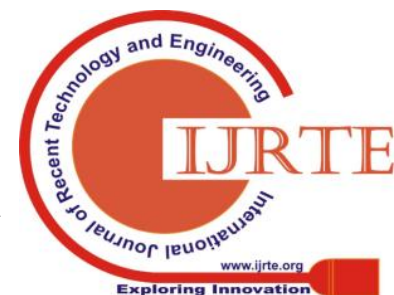


C functional groups are ascribed to the peak at $1541 \mathrm{~cm}^{-1}$.The absorbance bands at $1541,1238,1030$ and $780 \mathrm{~cm}-1$ are then contrasted with the flexing vibration of an $\mathrm{O}-\mathrm{H}$ in-plane, the asymmetric stretching of a C-O-C, the symmetric stretching of a C-O-C and the flexing vibration of an O-H out - of-plane. These peaks of absorption may improve in substances such ascyanidin 3-sambubioside, delphinidin 3-sambubioside, quercetin 3-rutinoside,etc. from polyphenolic compounds. In relation to the decrease, the intensity of an $\mathrm{O}-\mathrm{H}$ stretching and flexing vibrations reduces significantly in relation to the spectrum of S3.The change could be triggered by the reality that polyhydroxy groups of polyphenols are largely accountable for $\mathrm{Ag}$ (I) decrease and the appearance of Ag-O groups on the Ag-Nps surface [14 ].It can therefore be deduced that all these biomolecules are accountable for capping and stabilizing effectively. In the food, medicinal and cosmetics sectors, these environmentally friendly nanoparticles may find significance.

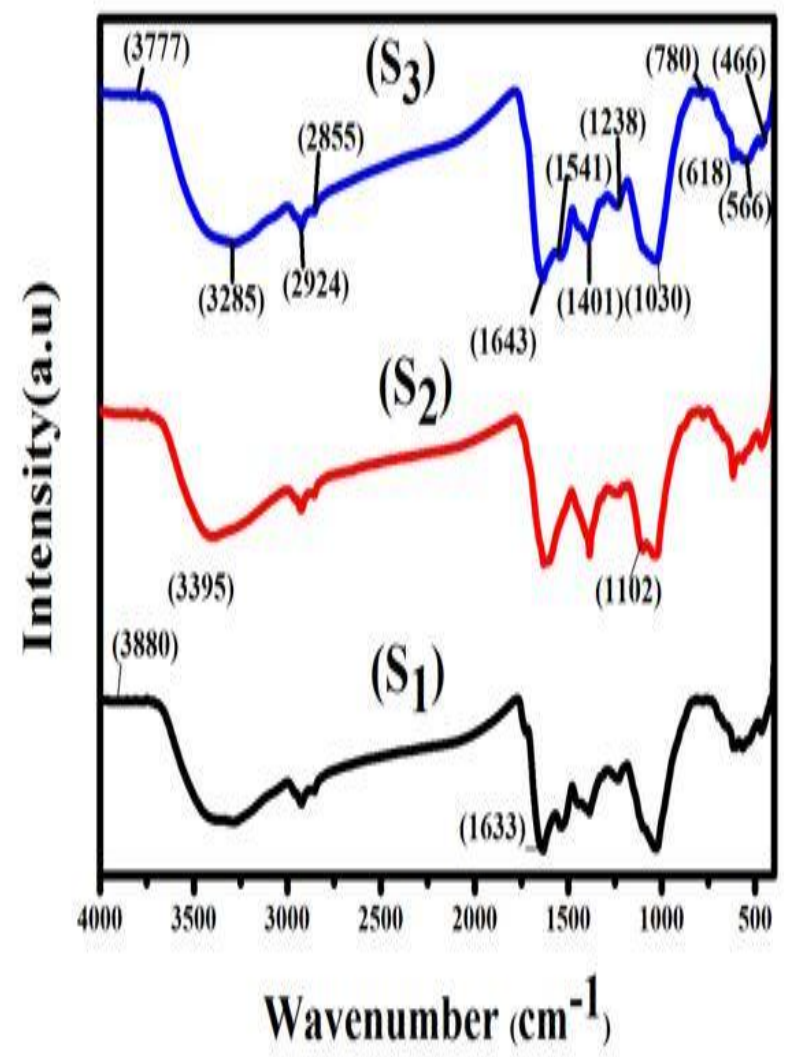

Fig. 2. FTIR analysis of Ag nanoparticles prepared with aqueous extractof

$\mathrm{S}_{1}, \mathrm{~S}_{2}$ and $\mathrm{S}_{3}$

SEM Analysis:The Ag-Nps morphology extracts S1, S2 and $\mathrm{S} 3$ were recognized via SEM pictures (Fig. 3a, c, and e).Most of the particles were spherical, and as an $\mathrm{Ag}-\mathrm{Nps}$, few are oval. Green synthetic Ag-Nps significantly enriched in the solution. The size of few chosen produced nanoparticles by SEM images was 7-15 nm. Most of the Ag-Nps had a diameter of $7.13 \mathrm{~nm}$. SEM images show the biomolecules of an Ag-Nps biosynthesizing. This layer brought plant extract in the stability and synthesis of green Ag NPs as a function of metabolites. The outcomes were achieved in agreement [15].Elementary deposited assessment of synthesized Ag-NPs was performed with EDX. EDX images of the test S1, S2 and $\mathrm{S} 3$ (Fig. 3 b, d, and f) show potent silver metal peaks in each sample as examined by Ag-NPs. Strong peaks of metallic nanoparticles appeared to be the $2.9 \mathrm{keV}$ in each of the EDX images (Fig. 3b, d, f), showing the existence of silver as an significantnanoparticlescomponent[16].

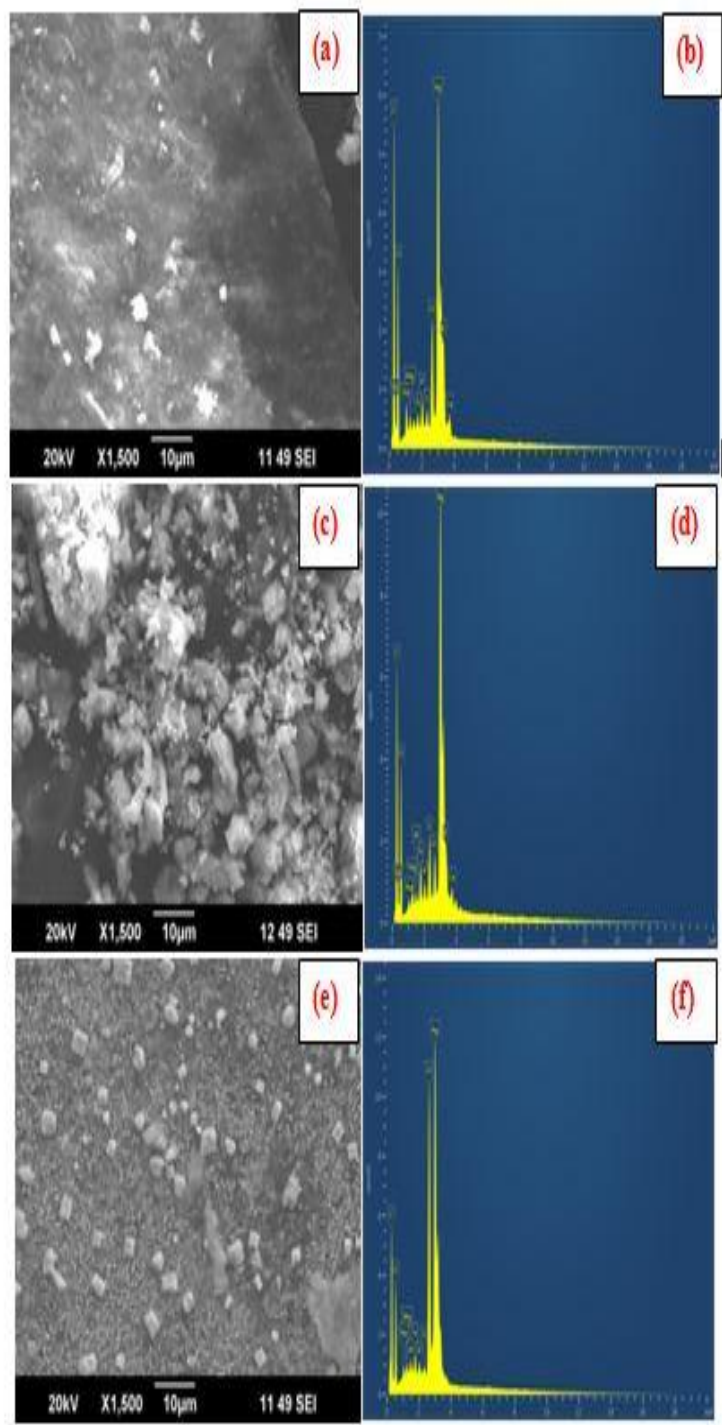

Fig. 3. SEM and EDAX analysis of Ag nanoparticles prepared with aqueous extractof $\mathrm{S}_{1}, \mathrm{~S}_{2}$ and $\mathrm{S}_{3}$

\section{UV-Vis absorption spectrum}

Ag-Nps samples of UV-Vis absorption spectrum S1, S2 and $\mathrm{S} 3$ are shown in Fig.4.The UV-Vis spectra found a broad spectrum curve in the shape of the bell. The broad plasmon band is generated by different plant extract metabolites created for application because they can also be shown in this spectrophotometric spectrum. Silver plasmon surface resonance (SPR) occurred at $450 \mathrm{~nm}$. This peak has risen to 4 hours with time.The spherical nanoparticles were shown only as a specified SPR set from the Mie theory. By enhancing the variety of particle shapes, the number of peaks increases[17].Then it is found that biosynthesized Ag-Nps is widely shape.$$
\text { shape. }
$$

in

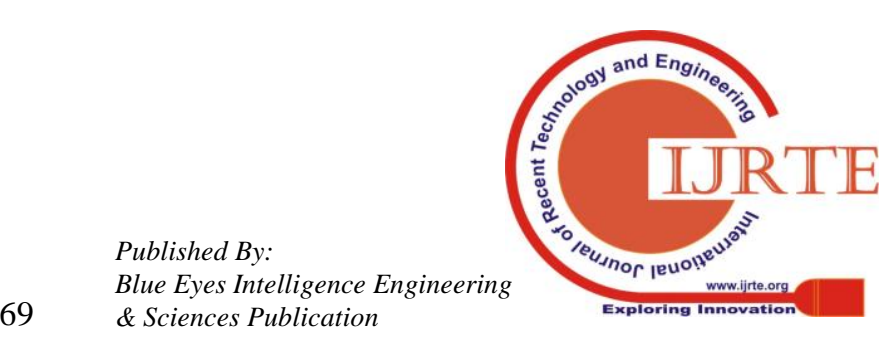



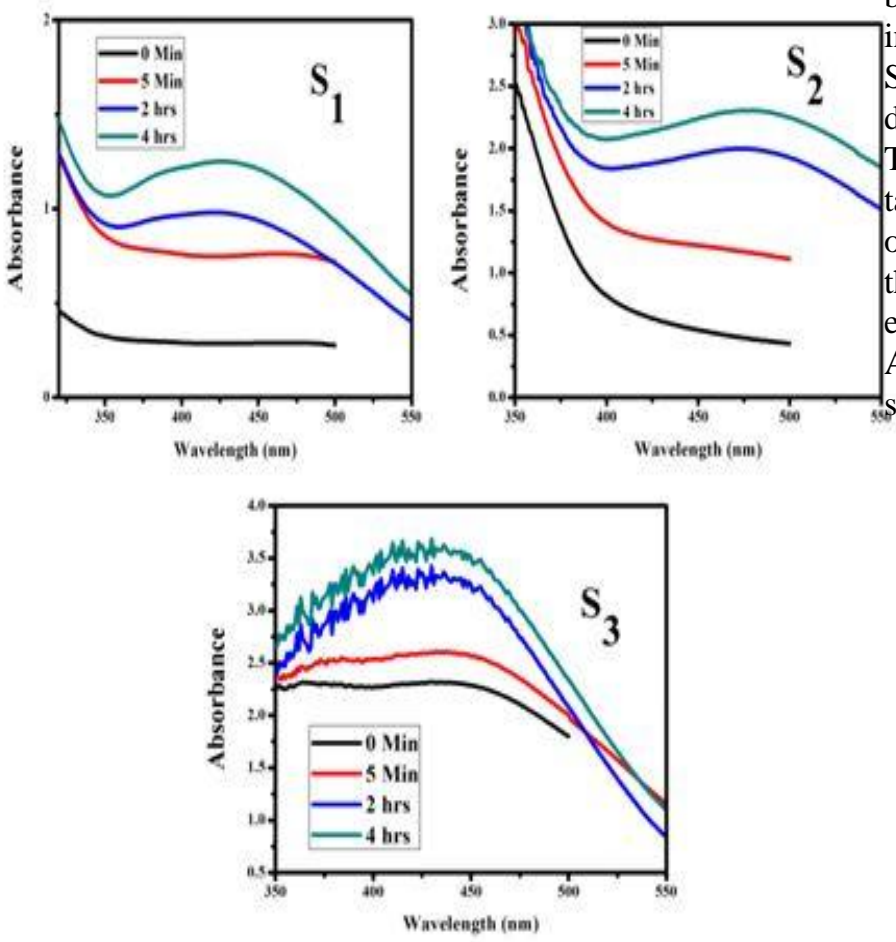

Fig. 4. UV spectrum analysis of Ag nanoparticles prepared with aqueous extractof $S_{1}, S_{2}$ and $S_{3}$

Analysis of dynamic light scattering. A well-organized technique for measuring particle diameters in the initial grain size distribution after response is the dynamic light scattering method[18 ].The primary factors affecting the Ag-Nps size allocation are numerous variables such as temperature, reaction time, and $\mathrm{pH}$ value. Fig. 5A showed the average particle size of the produced green $\mathrm{Ag}-\mathrm{Nps}$ silver nitrate solution in $\mathrm{S} 1$.Figure $5 \mathrm{~b}$ shows the average grain size of the $\mathrm{Ag}-\mathrm{Nps}$ when sampling S2.It is possible to see the volume of $\mathrm{Ag}-\mathrm{Nps}$ prepared with $\mathrm{S} 2$ as a reduction from 100 to $48 \mathrm{~nm}$. Fig. 5c showed the average particle size of Ag-Nps synthesized using S3 silver nitrate solution as a reduction of 100 to $78 \mathrm{~nm}$.
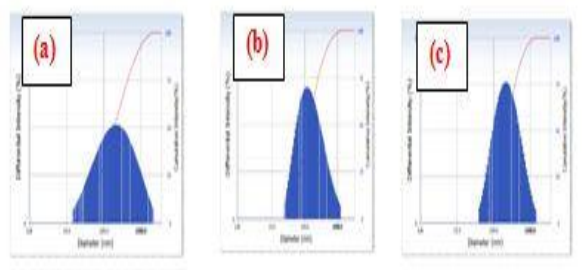

Fig. 5. Dynamic Light Scattering analysis of Ag nanoparticles prepared with aqueous extractof

$$
\mathrm{S}_{1}, \mathrm{~S}_{2} \text { and } \mathrm{S}_{3}
$$

\section{Plant-based antibacterial activity of AgNPs.}

Pathogenic infectious diseases continue to pose a severe risk to public health and societies. Since prehistory[19], plants and compounds derived from plants have been used to determine this worldwide issue. Green Ag-Nps synthesis was evaluated by disk diffusion method for antibacterial activity against pathogenic bacteria. Silver ion and silver compounds are poisonous to pathogens because they are extremely efficient in micro-organisms and have a big surface area. There were powerful antimicrobial activities in both Silver ions and Ag-Nps. The antibacterial activity of $\mathrm{S} 1, \mathrm{~S} 2$ and S3 samples comprising Ag-Nps showed that distinct alternatives with varying extent inhibited bacteria. The results of the antibacterial analysis are depicted in table-1. The prior research [ 20,21] agreed on this outcome.S3, which had the highest UV absorption denoting the development of high Ag-Nps quantities, had the greatest effect on all bacteria. The bacteria's dissimilarity to the Ag-Nps was owing to to variability in their membrane structure's thickness and components[22 ].

Table 1.Ag nanoparticles prepared with aqueous extract of $\mathrm{S} 1, \mathrm{~S} 2$ and S3 antibacterial

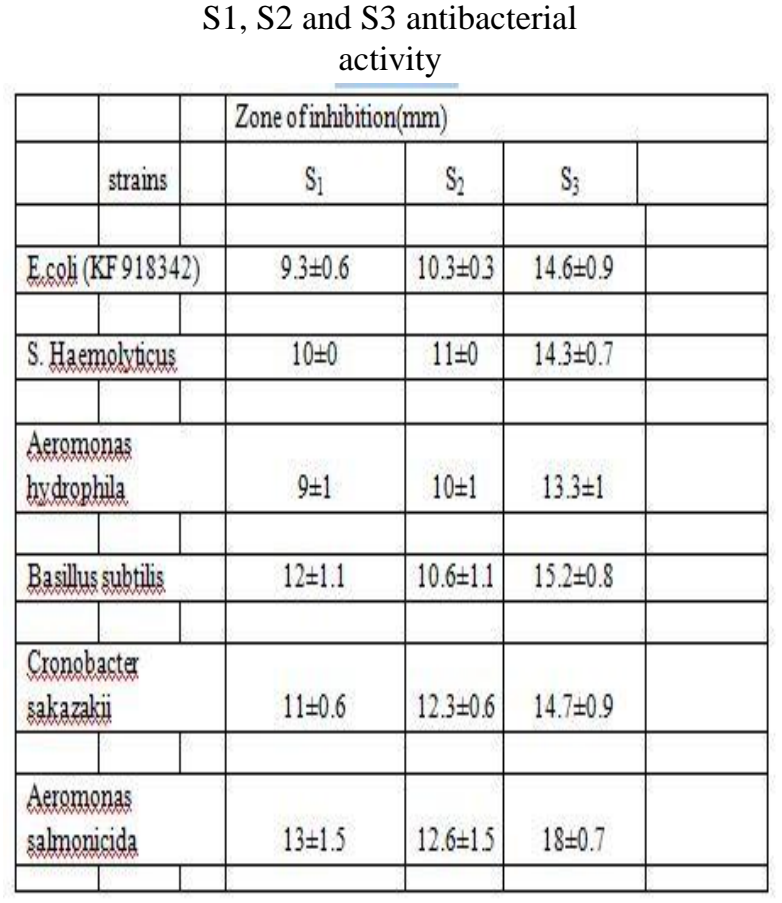

\section{CONCLUSION}

From our findings, we have for the first time a study of the plant sample of Chrysopogon zizanioid (S1), Ocimum sanctum (S2) and blended samples (S3) on the green synthesis of the 7 to $15 \mathrm{~nm}$ diameter highly steady spherical Ag-Nps. UV-vis, SEM, XRD and FTIR evaluated by various devices outlined the nanoparticles. Biosynthesized Ag-Nps ' bactericidal activity' mechanism shows its inhibitory role against different bacterial pathogens. This study recognized possible functional groups and effective compounds to reduce silver ions. Here we concluded that the $\mathrm{S}_{3}$ sample appeared a promising candidate for Ag-Nps synthesis and antibacterial activity.

\section{REFERENCES}

1. Dos Santos, C.A., Seckler, M.M., Ingle, A.P., Gupta, I., Galdiero, S., Galdiero, M., Gade, A., and Rai, M., J. Pharm. Sci., 2014, vol. 103, pp. $1931-1944$

2. Narayanan, K.B., and Sakthivel, N.,Adv. Colloid Interface Sci., 2010, vol. 156, pp. 1-13.

3. Raveendran, P., Fu, J., and Wallen, S.L., Green Chem., 2006, vol. 8, pp.34-38.

4. Armendariz, V., Gardea-Torresdey, J.L., Jose Yacaman, M., Gonzalez, J., Herrera, I., and Parsons, J.G., 2002. 
5. Song, J.Y., and Kim, B.S., Bioprocess Biosyst Eng., 2008, vol. 32, pp. 79-84.

6. Liz-Marzan, L.M., and Lado-Tourino, I., Langmuir., 1996 , vol. 123, pp. 585-3589.

7. Esumi, K., Tano, T., Torigoe, K., and Meguro, K., J Chem Mater., 1990, vol. 2 ,

pp. 564-567.

8. Pileni, M.P., Pure Appl Chem., 2000, vol. 72, pp. 53-65.

9. Sun, Y.P., Atorngitjawat, P., and Meziani, M.J., Langmuir., 2001, vol. 17 , pp. $5707-5710$

10. Henglein, A., J Phys Chem B., 1993, vol. 97, pp.5457-5471.

11. Henglein, A., J Chem Mater., 1998, vol. 10, pp. 444-446.

12. Henglein, A., Langmuir., 2001, vol. 17, pp. 2329-2333.

13. Klaus, T., Joerger, R., Olsson, E., and Granqvist, C.G., J Proc Natl Acad Sci USA., 1999, vol. 96, pp.13611-13614.

14. Nair, B., and Pradeep, T., Cryst Growth Des., 2002, vol. 2, pp.293-298.

15. Willner, I., Baron, and R., Willner, B., J Adv Mater., 2006, vol. 18, pp.1109-1120.

16. Ankamwar, B., Gharge, M., and Sur, U.K., Adv. Sci. Eng. Med., 2015, vol. 7 , pp. $480-484$

17. Spectrosc., 2015, vol. 134, pp.310-5.

18. Ulug, B., HalukTurkdemir, M., Cicek, A., and Mete, A., Spectrochim Part A. Mol Biomol Spectrosc., 2015, vol.135, pp.153-61.

19. Jacob, J., Mukherjee, T., and Kapoor, S., Mater. Sci. Eng. C., 2012, vol. 32, pp. $1827-1834$

20. Gopal Suresh, Poosali Hariharan Gunasekar, Dhanasegaran Kokila, Durai Prabhu, Devadoss Dinesh, Nagaiya Ravichandran, Balasubramanian Ramesh, and Arunagirinathan Koodalingam, Ganesan Vijaiyan Siva. Spectrochim. Acta, Part A., 2014, vol. 127, pp.61-66.

21. Kasthuri, J., Veerapandian, S., and Rajendiran, N., Colloids Surf. B., 2009 , vol. 68

pp.55-60.

Oves, M., Aslam, M., Rauf, M.A., Qayyum, S., Qari, H.A., Khan, M.S., and Alam, M.Z., Mater. Sci. Eng. C., 2018, vol. 89, pp. $429-443$. 